\title{
Prospecção científica e tecnológica do uso da vibração focal em comprometimentos motores em decorrência de patologias neurológicas
}

Scientific and technological prospection of the use of focal vibration in motor commitments due to neurological pathologies

Prospección científica y tecnológica del uso de la vibración focal en compromisos motorizados por patologías neurológicas

Tassiane Maria Alves Pereira ORCID: https://orcid.org/0000-0002-8876-7438 Universidade Federal do Piauí, Brasil E-mail: tassiane.alves07@gmail.com

Jéssica Inara de Brito Siqueira ORCID: https://orcid.org/0000-0001-8279-1050 Universidade Federal do Piauí, Brasil E-mail: jessicainarabrito@hotmail.com

Lucas da Silva Nascimento ORCID: https://orcid.org/0000-0002-6087-096X Universidade Federal do Piaú, Brasil E-mail: lucas_silvaphb@hotmail.com

Cassio Costa Almeida ORCID: https://orcid.org/0000-0001-9041-0887 Universidade Federal do Piauí, Brasil E-mail: jcassiocosta@gmail.com

Marco Orsini

ORCID: https://orcid.org/0000-0002-8526-6937

Universidade Iguaçu, Brasil

E-mail: orsinimarco@hotmail.com

Janaína de Moraes Silva

ORCID: https://orcid.org/0000-0002-8693-3957 Universidade Estadual do Piauí, Brasil

E-mail: fisiojanainams@gmail.com

Jefferson Almeida Rocha

ORCID: https://orcid.org/0000-0001-6619-2293 Universidade Federal do Maranhão, Brasil

E-mail: jeffersonbiotec@gmail.com

Victor Hugo do Vale Bastos

ORCID: https://orcid.org/0000-0001-7425-4730 Universidade Federal do Piauí, Brasil E-mail: victorhugobastos@ufpi.edu.br

\section{Resumo}

O Brasil apresenta uma prevalência de 1,3\% deficientes físicos. No que tange os comprometimentos físicos relacionados a patologias neurológicas, estes geram incapacidade funcional em todo o mundo. Dessa forma, este trabalho teve como objetivo a realização da prospecção científica e tecnológica de pesquisas sobre uso da vibração focal associada a dispositivos móveis vestíveis em indivíduos com comprometimentos motores decorrentes de patologias neurológicas. Na busca por patentes foram usadas as bases: Espacenet, INPI, USPTO e Web of Science; e os artigos nas bases: Scielo, Scopus, Science Diretc, Web of Science, Pubmed, de 1967 até 2020. Os resultados da busca por patentes no contexto mencionado foram negativos, e em relação aos artigos, obteve-se resultados significativos com 667 publicações no Science Diretc com pico de publicação no ano de 2018. Observou-se então, que novos estudos devem ser realizados para fomentar as propriedades e eficácia da vibração focal, e promover incentivos ao desenvolvimento e produções tecnológicas e científicas.

Palavras-chave: Vibração; Reabilitação; Dispositivos eletrônicos vestíveis.

\footnotetext{
Abstract

Brazil has a prevalence of $1.3 \%$ disabled people. Regarding the physical impairments related to neurological pathologies, they generate functional disability worldwide. Thus, this work aimed to carry out scientific and technological research on research on the use of focal vibration associated with wearable mobile devices in individuals
} 
with motor impairments resulting from neurological pathologies. In the search for patents the bases were used: Espacenet, INPI, USPTO and Web of Science; and articles in the databases: Scielo, Scopus, Science Direct, Web of Science, Pubmed, from 1967 to 2020 . The results of the search for patents in the mentioned context were negative, and in relation to the articles, significant results were obtained with 667 publications in the Science Direct with a peak of publication in 2018. It was observed then, that new studies must be carried out to promote the properties and effectiveness of the focal vibration, and to promote incentives to the development and technological and scientific productions.

Keywords: Vibration; Rehabilitation; Wearable electronic devices.

\section{Resumen}

Brasil tiene una prevalencia del $1.3 \%$ con discapacidades físicas. En cuanto a las deficiencias físicas relacionadas con patologías neurológicas, estas generan discapacidad funcional a nivel mundial. Así, este trabajo tuvo como objetivo realizar una investigación científica y tecnológica sobre la investigación sobre el uso de la vibración focal asociada a los dispositivos móviles portátiles en personas con deficiencias motoras derivadas de patologías neurológicas. En la búsqueda de patentes se utilizaron las bases: Espacenet, INPI, USPTO y Web of Science; y los artículos en las bases de datos: Scielo, Scopus, Science Director, Web of Science, Pubmed, de 1967 a 2020. Los resultados de la búsqueda de patentes en el contexto mencionado fueron negativos, y en relación a los artículos se obtuvieron resultados significativos con 667 publicaciones en el Directorio de Ciencias con un pico de publicación en 2018. Luego se observó que se deben realizar nuevos estudios para promover las propiedades y efectividad de la vibración focal, y promover incentivos para el desarrollo y producciones tecnológicas y científicas.

Palabras clave: Vibración; Rehabilitación; Dispositivos electrónicos portátiles.

\section{Introdução}

De acordo com o Censo do Instituto Brasileiro de Geografia e Estatística (IBGE) realizado em 2010 com releitura no ano de 2013, a Pesquisa Nacional de Saúde (PNS) estimou que de 200,6 milhões de pessoas residentes em domicílios brasileiros, cerca de 6,2\% possuía pelo menos uma das quatro deficiências (auditiva, visual, física e intelectual), sendo uma prevalência de $1,3 \%$ de deficiência física, nas quais $46,8 \%$ possuía grau intenso ou muito intenso de limitações, ou ainda não conseguia realizar as atividades habituais (Ministério da Saúde [MS], 2019).

Os comprometimentos motores ocasionadas por patologias neurológicas são uma das principais causas de incapacidade funcional no mundo, isso devido as alterações decorrentes de danos cerebrais que afetam principalmente a área motora, provocando déficits importantes que prejudicam a independência e funcionalidade destes pacientes. Dentre as alterações provenientes dessas condições patológicas, pode-se observar distúrbios de movimentos, espasticidade, fraqueza muscular, déficits de equilíbrio, marcha e sensibilidade comuns nestas patologias (Khalifeloo et al., 2018; Li et al., 2019a; Costantino, Galuppo, \& Romiti, 2016).

Estudos revelaram que em patologias neurológicas como o Acidente Vascular Encefálico (AVE), 80\% dos pacientes requerem tratamento na extremidade superior, com $40 \%$ deles apresentando deficiências de moderadas a graves neste segmento. Sendo o membro superior o responsável por grande maioria das atividades de vida diária desenvolvidas, estes pacientes acabam apresentando redução da funcionalidade e consequentemente prejuízos na sua qualidade de vida (Alashram, Padua, Romagnoli, \& Annino, 2019).

Alguns recursos terapêuticos estão envolvidos na reabilitação neurofuncional que visam essencialmente proporcionar melhora da condição motora e da funcionalidade desses pacientes, nos quais podemos citar a imagética motora (Hanson \& Concialdi, 2019; Bruno, Fossataro, \& Garbarini, 2018), terapia espelho (Costa, Silveira, Clementino, Borges, \& Melo, 2016; Medeiros, Fernandes, Lopes, Cacho, \& Cacho, 2014), Facilitação Neuromuscular Proprioceptiva (Pola, Bergmann, \& Silva, 2015; Lacerda, Gomes, \& Pinheiro, 2013), realidade virtual (Deutsch, 2011; Pompeu, Alonso, Masson, Pompeu, \& TorrianiPasin, 2014; Galvão et al., 2015), vibração focal (Alashram et al., 2019; Li et al., 2019b; Toscano et al., 2019), entre outras.

Especificadamente, a vibração focal é uma técnica que aplica um estímulo vibratório ao músculo ou tendão gerando entradas aferentes Ia e, consequentemente ativação das terminações primária do fuso muscular, levando a alterações nas vias 
corticoespinais e aumento da excitabilidade do córtex motor primário, nas quais produzem modificações no comprimento da fibra muscular, aumento da força e desempenho muscular (Alashram et al., 2019; Guang, Ji, \& Shi, 2018; Li et al., 2019a; Zinke, Gebel, Granacher, \& Prieske, 2019). A vibração apresenta características variáveis como frequência, amplitude das ondas, tipo de equipamento usado, tempo de exposição, posição do membro e estado de repouso ou contração muscular que podem levar a alterações distintas durante o treinamento (Jordan, Norris, Smith, \& Herzog, 2005; Kitazani \& Griffin 1998; Martin \& Park, 1997; Kojima et al., 2018).

Estudos apontaram que o uso da vibração focal através de alguns dispositivos vestíveis empregados no membro superior como Remind-to-Move (Wei, Fong, Chung, Cheung, \& Chow, 2019), TheraBracelet (Lakshminarayanan, Wang, Webster, \& Seo, 2017), WAVES (Da-Silva et al., 2019), VibroSleeve (Prabhu, Hasan, Wise, MacMahon, \& McCarthy, 2020) apresentaram melhoras significativas ao membro superior acometido por condições patológicas neurológicas. Acredita-se que a estimulação sensorial periférica impulsiona mudanças no córtex motor primário, aumentando a excitabilidade cortical e por consequência a funcionalidade motora do membro (Seo et al., 2019).

Os dispositivos móveis vestíveis vêm sendo utilizados na reabilitação neurofuncional tanto como auxílio no diagnóstico, podendo reduzir o tempo de avaliação e fornecer dados objetivos, de tal forma que possa quantificar e/ou graduar sobre as capacidades ou habilidades desempenhadas pelo paciente, assim como no processo de reabilitação tornando mais acessível e atraente o tratamento (Maceira-Elvira, Popa, Schmid, \& Hummel, 2019; Da-Silva et al., 2019).

Diante disso, o objetivo deste trabalho é realizar uma prospecção científica e tecnológica por meio das publicações de artigos e monitoramento de documentos de patentes respectivamente, que utilizaram dispositivos móveis vestíveis associados a vibração em indivíduos com comprometimentos motores decorrentes de patologias neurológicas, a modo de apresentar novas possibilidades de reabilitação neurofuncional.

\section{Metodologia}

O presente artigo trata-se de um estudo de prospecção científica e tecnológica. Segundo Amparo, Ribeiro, \& Guarieiro (2012) e Santos, Coelho, Santos, \& Fellows Filho (2010), as metodologias de prospeç̧ão são ferramentas úteis para o levantamento do estado da arte e tem como objetivo a construção do conhecimento. Para a realização da pesquisa, realizou-se buscas em bancos de dados de patentes para o estudo prospectivo tecnológico e em bases de dados nacionais e internacionais para realização da prospecção científica.

Para a realização da prospecção científica foi feita uma busca nas bases de dados: Scientífic Eletronic Library Online (Scielo), Nacional Center for Biotechnology information (PubMed), Thompson Reuters (Web of Science), grupo Elsevier Group (Scopus) e Science Direct. Os descritores utilizados foram: wearable electronic device and vibration and rehabilitation. A busca foi feira considerando título/resumo (title/abstract) e todas os termos foram usados em português e inglês de acordo com a base.

A prospeç̧ão de patentes foi realizada nas plataformas: Instituto Nacional da Propriedade Industrial (INPI) Europe's Network of Patents databases (Espacenet), United States Patent and Trademark Office (USPTO) e Thompson Reuters (Web of Science) com a busca das palavras-chave: wearable electronic device and vibration and rehabilitation, sem restrições e sem limite de datas de publicação.

Os dados prospectados foram obtidos levando em consideração todos os artigos publicados e/ou registros de patentes até dezembro de 2020. Após o processo de busca, foi realizado o tratamento dos dados obtidos para obter melhor visualização e compreensão dos resultados. A figura 1 apresenta o organograma das bases de dados utilizadas com seus respectivos descritores. 
Figura 1 - Organograma das bases utilizadas nas prospecções científicas e tecnológicas.

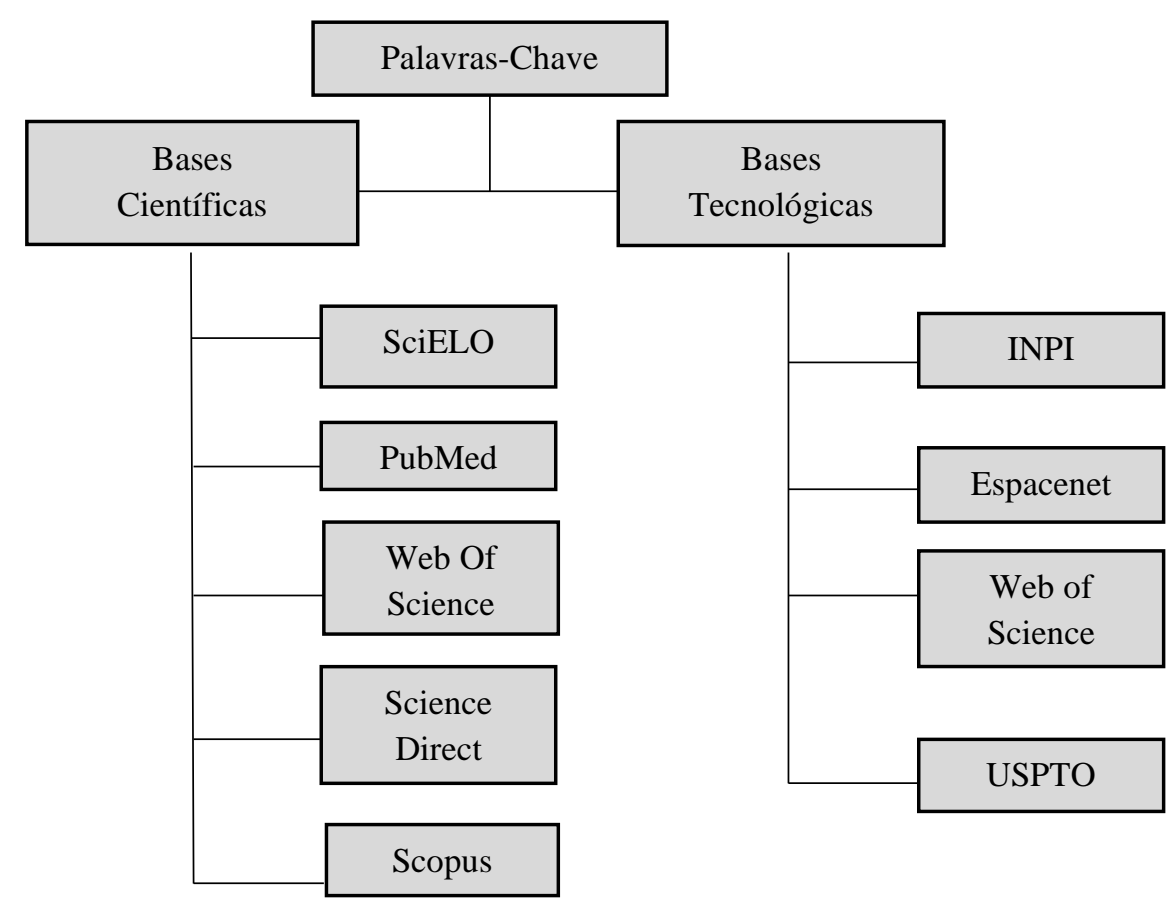

Fonte: Elaborado pelos autores deste artigo (2020).

\section{Resultados e Discussão}

Este estudo permite elucidar as publicações científicas e tecnológicas acerca da utilização de dispositivos móveis vestíveis associados a vibração para reabilitação de pacientes com comprometimentos motores ocasionado por patologias neurológicas. Com isso, pode-se observar que há uma desproporção entre o número de publicações científicas e a quantidade de patentes registradas.

\subsection{Prospecção Tecnológica}

A busca por patentes relacionadas ao tema exposto não evidenciou registros positivos (Tabela 1), o que se pode associar a pequena produção de dispositivos vibratórios auxiliares a reabilitação neurofuncional e/ou a necessidade de estudos que demonstre os efeitos positivos da ferramenta e que apresente propriedades patenteáveis.

Tabela 1 - Depósitos de patentes sobre vibração associada a dispositivos móveis vestíveis no processo de reabilitação pesquisados nas bases de dados da INPI, Espacenet, USPTO e Web of Science e seus respectivos descritores.

\begin{tabular}{ccccc}
\hline Palavras-chave & Espacenet & USPTO & Web of Science & INPI \\
\hline $\begin{array}{c}\text { Wearable electronic device and vibration } \\
\text { and rehabilitation }\end{array}$ & 0 & 0 & 0 & 0
\end{tabular}

Fonte: Elaborado pelos autores deste artigo (2020).

Em países de primeiro mundo, as patentes são frequentemente utilizadas pelas empresas inovadoras como ferramentas para impedir plágios pelos adversários. Isso é destacado pela quantidade registrada através de um levantamento, realizado entre 
os 20 maiores escritórios de concessão de patentes mundial, utilizando dados do ano de 2013, indicam os Estados Unidos em primeiro lugar, com 2,3 milhões de patentes, seguido do Japão, que tem 1,8 milhão. Depois estão China (1 milhão), Coreia do Sul (812 mil), Alemanha (569 mil), França (500 mil), Reino Unido (469 mil). O Brasil está na 19ª posição, com 41.453 patentes válidas (Arnold \& Dos Santos, 2016).

No Brasil, o desenvolvimento de patentes teve um marco regulatório importante com a criação da Lei de Inovação (Lei 10.973 de 02 de dezembro de 2004), que estabeleceu incentivos à inovação e à pesquisa científica no ambiente produzindo, proporcionado autonomia tecnológica e ao desenvolvimento industrial do país. Além disso, fatores como a consciência da importância do patenteamento no meio acadêmico, criação da Rede de Propriedade Intelectual, Cooperação, Negociação e Comercialização de Tecnologia, a criação do Fórum Nacional de Gestores de Inovação e Transferência de Tecnologia, a disponibilização de recursos para a criação e consolidação dos núcleos de inovação tecnológica e a inclusão de cláusulas relativas aos direitos de propriedade intelectual em projetos financiados por órgãos públicos envolvendo parcerias com empresas contribuíram para a criação e consolidação dos núcleos de inovação tecnológica e para o aumento do número de depósitos de patentes de universidades e institutos de pesquisa no Brasil (Souza, Basbarfene, \& Pereira, 2011).

Apesar das regulamentações, ainda existem alguns desafios para desenvolvimento de patentes que estão relacionados com a busca por acrescentar credibilidade ao produto e contribuir para o conceito de uma empresa inovadora, a intenção de monopolizar a patente com o intuito de impedir terceiros a fabricarem, usarem, venderem ou importarem a invenção, a proteção financeira garantindo que o rendimento seja superior ao investimento, e principalmente, o tempo de processamento de um pedido de patente junto ao órgão responsável (Ferreira, Guimarães, \& Contador, 2009).

É importante ressaltar que de acordo com INPI as patentes devem obedecer a requisitos que os torne patenteáveis. Requisitos como novidade, atividade inventiva e aplicação industrial devem nortear todo o processo de planejamento e construção de uma patente, sendo o quesito novidade o que tem influência direta na comunicação científica e tecnológica. Patentes do tipo Invenção tem prazo de validade de 20 anos, e o modelo de utilidade de 15 anos (Mueller \& Peruchi, 2014).

Além disso, no meio acadêmico brasileiro, a Propriedade Intelectual ainda é desconhecida e pouco propagada, a situação se agrava ainda mais quando confrontamos os números de investimentos em pesquisas pelas universidades brasileiras com a falta de comprometimento dos pesquisadores com a proteção dos trabalhos produzidos por meio de patentes e sua inserção no mercado e na sociedade. Com isso, as tecnologias aqui produzidas padecem por falta de proteção, o que acarreta sua não utilização em fins comerciais e como fonte de informações importantes em outras pesquisas (Motta \& Pereira, 2019).

Independente dos desafios encontrados neste processo, as patentes ocupam um espaço significativo nas discussões científicas. Com a possibilidade de geração de um produto, os pesquisadores ampliam suas oportunidades e além de atingir o reconhecimento de suas descobertas, também podem comercializá-las. Assim, pode-se considerar a grande importância da produção de patentes e evolução do campo tecnológico e científico (Moura, Lago, Mesquita, \& Alves, 2014; Paranhos \& Ribeiro, 2018).

Diante dos fatores expostos, torna-se necessário a mudança desse cenário, visando o incentivo de produção científica e tecnológica, através de mudanças nas políticas de incentivo, fomento das pesquisas e desenvolvimentos, e em prol da praticidade e diminuição dos prazos de registro de depósitos e concessão de patentes. Dessa forma, as produções contribuirão tanto para a comunidade acadêmico-científica quanto na solução de problemas pertinentes a sociedade.

\subsection{Prospecção Científica}

A prospecção científica apresentou a quantidade de 798 artigos publicados relacionados ao tema abordado de acordo com as bases apresentadas na Tabela 2, mostrando-se discrepante em relação à tecnológica. 
Research, Society and Development, v. 10, n. 5, e13310514736, 2021

(CC BY 4.0) | ISSN 2525-3409 | DOI: http://dx.doi.org/10.33448/rsd-v10i5.14736

Tabela 2 - Quantidade de artigos encontrados e seus respectivos descritores.

\begin{tabular}{ccc|c|c|c}
\hline Palavras-chave & PubMed Scopus & Web of Science & Science Direct & Scielo \\
\hline $\begin{array}{c}\text { Wearable electronic device and vibration } \\
\begin{array}{c}\text { and rehabilitation } \\
\text { a }\end{array}\end{array}$ & 116 & 0 & 667 & 0 \\
\hline
\end{tabular}

Fonte: Elaborado pelos autores deste artigo (2020).

Com relação aos resultados, vale destacar que embora se tenha uma quantidade numérica significativa de artigos, muitos se diferem por apresentar só um termo/descritor, o que não compreende em sua totalidade à artigos que trazem evidências sobre dispositivos móveis vestíveis que empregam a vibração para tratamento de pacientes com comprometimentos motores patológicos.

Observou-se também que as produções expressaram um pico no ano de 2018 em todas as bases (Figura 2) e seguiu crescente nos anos seguintes, principalmente as publicações da base Science Direct (Figura 2.C), o que pode se considerar que existem e estão sendo elaboradas pesquisas a respeito do tema estudado, e principalmente reformulando inovações já existentes, dando um caráter mais moderno e ainda, com baixo custo (Lakshminarayanan et al., 2017).

Figura 2 - Gráficos da relação entre anos de publicações e a quantidade de artigos publicados em cada base científica com seus respectivos descritores. (A) Scopus. (B)PubMed. (C)Science Direct.

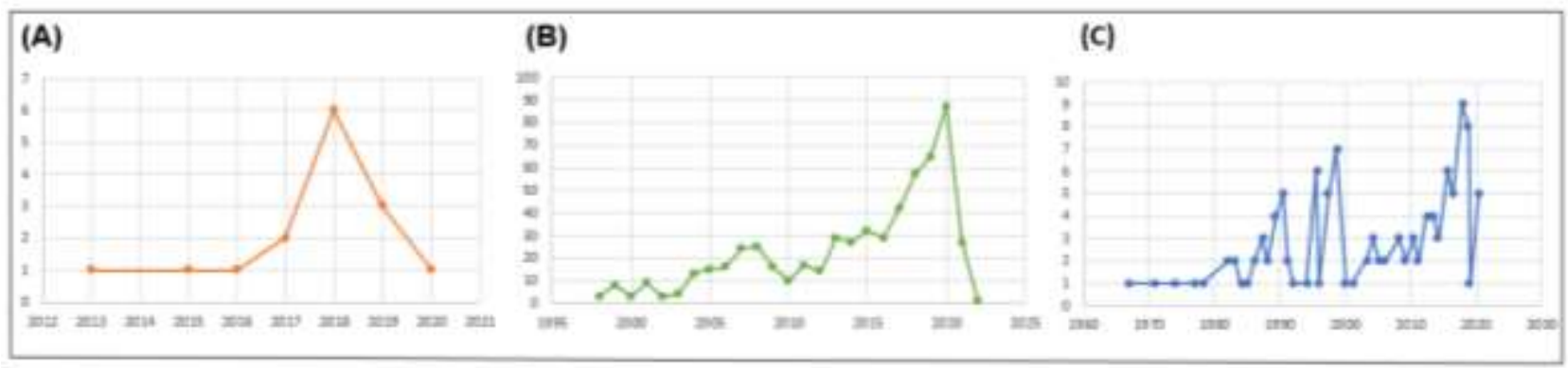

Fonte: Elaborado pelos autores deste artigo (2020).

Dos artigos científicos encontrados, poucos fazem referência a aplicação da vibração por meio de dispositivos vestíveis que favoreçam a total adesão da pele com os sensores vibrotáteis e permitam intensificar a ação da vibração na musculatura explorada. Estes dispositivos móveis vestíveis são descritos como dispositivos utilizados como acessórios que transmitem dados, acompanham funções e acessa à Web permitindo conexões virtuais com o usuário (Cantanhede, Gammarano, Dias, \& Arruda Filho, 2018).

Para Da-Silva et al., (2019), o desenvolvimento de órtese vestível associada a vibração que monitora e fornece feedback "ao vivo" da atividade dos membros superiores, para lembrar os pacientes de usar o braço de braçada em atividades diárias, abriu uma lacuna importante na base de evidências, que pode gerar uma viabilidade para o desenvolvimento de tecnologias emergentes e acessíveis para ajudar esses pacientes com comprometimentos motores.

Na reabilitação neurofuncional, estes dispositivos proporcionaram um feedback sensorial que fez com que os usuários se adequem e utilizem o equipamento. Quanto as repercussões neurofisiológicas, estudos comprovaram que a utilização destes dispositivos associados a vibração facilitou a função da mão e/ou do membro superior comprometido deferindo um maior grau de liberdade e funcionalidade (Wei et al., 2019; Lakshminarayanan et al., 2017; Da-Silva et al., 2019; Prabhu et al., 2020).

A geração de conhecimento e tecnologia é responsabilidade da ciência, a propagação do saber é fomentada pela 
divulgação e produção de desfechos de pesquisas científicas. A divulgação de resultados de pesquisas científicas influencia positivamente no crescimento e na difusão do conhecimento científico. Para isso, vale ressaltar o importante papel do pesquisador como mediador desse processo de comunicação científica ao passo que o mesmo disponibiliza os resultados de suas pesquisas para a comunidade, ao contrário disso, as pesquisas não divulgadas não são reconhecidas com pesquisas com algum valor útil, pois poderão ser apreciadas pela crítica da comunidade científica (Angerami \& Almeida, 1982).

Diante das evidências expressas sobre a vibração e as repercussões funcionais que esta desempenha, é notável a necessidade de investimentos e pesquisas que possam intervir no processo de reabilitação neurofuncional. Mediante isso, a prospecção intensifica essas limitações e fortalece um posicionamento crítico sobre a carência de produções neste âmbito.

\section{Conclusão}

O Brasil apresenta um número crescente de pessoas acometidas por doenças neurológicas e que possuem deficiência física. Diante deste cenário, é necessário que haja recursos terapêuticos que promovam a reabilitação neurofuncional.

Com base no que foi exposto, ainda não existem patentes que resguardam produções tecnológicas empregadas neste contexto relacionadas ao uso de vibração focal. As produções científicas apontam resultados satisfatórios da intervenção no membro superior acometimento capaz de promover ativação cortical e consequentemente ganhos motores significativos e melhora da funcionalidade. No entanto, são necessárias mais pesquisas com a utilização de dispositivos móveis vestíveis contribuindo no processo de reabilitação física.

Vale ressaltar a importância dos estudos de prospecção e monitoramento informacional que permitem mapear todos os conhecimentos iniciais e essenciais à competitividade de um determinado setor, permitindo encontrar o que se tem nas bases de dados e colaborar para a criação de novos estudos, a partir das lacunas encontradas proporcionado novas oportunidades de pesquisa e desenvolvimento científico e tecnológico.

Conclui-se que foi possível compreender que ainda há muitos caminhos a seguir relacionados à utilização da vibração focal, enfocando tanto em produções de novos estudos como na construção de novos equipamentos, principalmente que apresentem propriedades patenteáveis, para que se possa aumentar as produções e alavancar o campo tecnológico e científico trazendo relevâncias a sociedade em geral.

Nesta perspectiva, novos estudos devem ser realizados para fomentar as propriedades e eficácia da vibração focal, e promover incentivos ao desenvolvimento de novos recursos com requisitos patenteáveis, que possam intensificar as produções, tanto científicas quanto tecnológicas e permitir a disseminação de conhecimentos e valorização do pesquisador, assim como de suas descobertas. Espera-se ainda que, as dificuldades encontradas no processo de pedido de patente sejam corrigidas e/ou amenizadas, tornando-as mais viáveis e mais rápidas, podendo dessa maneira, incentivar o desenvolvimento de mais produções, tendo em vista a facilidade de processamento e as expressivas contribuições que as patentes trazem ao meio tecnológico, destacando sempre também, o reconhecimento e valorização do pesquisador.

Contudo, é necessário o desenvolvimento de novas tecnologias implementadas com o uso de vibração na qual forneçam a oportunidades de novos recursos terapêuticos na reabilitação neurofuncional, nesta mesma vertente espera-se que estes estudos possam sugerir e proporcionar a criação de produtos patenteáveis promovendo o incentivo à produção tecnológica.

\section{Agradecimentos}

O presente trabalho foi realizado com apoio da Coordenação de Aperfeiçoamento de Pessoal de Nível Superior - Brasil (CAPES) - Código de Financiamento 001. 


\section{Referências}

Alashram, A. R., Padua, E., Romagnoli, C., \& Annino, G. (2019). Effectiveness of focal muscle vibration on hemiplegic upper extremity spasticity in individuals with stroke: A systematic review. Neurorehabilitation, 45(4), 471-481. https://pubmed.ncbi.nlm.nih.gov/31868686/.

Amparo, K. K. D. S., Ribeiro, M. D. C. O., \& Guarieiro, L. L. N. (2012). Estudo de caso utilizando mapeamento de prospecção tecnológica como principal ferramenta de busca científica. Perspectivas em Ciência da Informação, 17(4), 195-209. https://www.scielo.br/pdf/pci/v17n4/12.pdf.

Angerami, E. L. S., \& Almeida, M. C. P. D. (1982). Divulgação do conhecimento científico produzido na enfermagem. Revista da Escola de Enfermagem da USP, 16(2), 211-223. https://www.scielo.br/pdf/reeusp/v16n2/0080-6234-reeusp-16-2-211.pdf.

Arnold, F. R., \& dos Santos, C. B. (2016). A CONCESSÃO DE PATENTES NO BRASIL: UM ESTUDO EXPLORATÓRIO. Caderno PAIC, 17(1), 101-115. https://cadernopaic.fae.edu/cadernopaic/article/view/206/167.

Bruno, V., Fossataro, C., \& Garbarini, F. (2018). Inhibition or facilitation? Modulation of corticospinal excitability during motor imagery. Neuropsychologia, 111, 360-368. https://pubmed.ncbi.nlm.nih.gov/29462639/.

Cantanhede, L. R. C., Gammarano, I. D. J. L. P., Dias, E. J. P., \& Arruda Filho, E. J. M. (2018). Comportamento do consumidor de tecnologia vestível: características que influenciam na intenção de consumo. Revista Eletrônica de Administração, 24(3), 244-268. https://www.scielo.br/pdf/read/v24n3/1413-2311read-24-03-00244.pdf.

Costa, V. S., Silveira, J. C. C., Clementino, T. C. A., Borges, L. R. D. M., \& Melo, L. P. (2016). Efeitos da terapia espelho na recuperação motora e funcional do membro superior com paresia pós-AVC: uma revisão sistemática. Fisioterapia e Pesquisa, 23(4), 431-438. https://www.scielo.br/scielo.php?pid=S180929502016000400431\&script=sci_abstract\&tlng=pt.

Costantino, C., Galuppo, L., \& Romiti, D. (2017). Short-term effect of local muscle vibration treatment versus sham therapy on upper limb in chronic poststroke patients: a randomized controlled trial. Eur J Phys Rehabil Med, 53(1), 32-40. https://europepmc.org/article/med/27598342.

Da-Silva, R. H., Moore, S. A., Rodgers, H., Shaw, L., Sutcliffe, L., van Wijck, F., \& Price, C. I. (2019). Wristband Accelerometers to motiVate arm Exercises after Stroke (WAVES): a pilot randomized controlled trial. Clinical rehabilitation, 33(8), 1391-1403. https://pubmed.ncbi.nlm.nih.gov/30845829/.

Deutsch, J. E. (2011). Using virtual reality to improve walking post-stroke: translation to individuals with diabetes. Journal of Diabetes Science and Technology, 5(2), 309-314. https://pubmed.ncbi.nlm.nih.gov/21527098/.

Ferreira, A. A., Guimarães, E. R., \& Contador, J. C. (2009). Patente como instrumento competitivo e como fonte de informação tecnológica. Gestão \& Produção, 16(2), 209-221. https://www.scielo.br/scielo.php?pid=s0104-530x2009000200005\&script=sci_abstract\&tlng=pt.

Galvão, M. L. C., Gouvêa, P. M., Ocamoto, G. N., Silva, A. T., dos Reis, L. M., Kosour, C., \& Silva, A. M. (2015). Efeito da realidade virtual na função motora do membro superior parético pós-Acidente Vascular Cerebral. Revista Neurociências, 23(4), 493-498. http://www.revistaneurociencias.com.br/edicoes/2015/2304/originais/1038original.pdf.

Guang, H., Ji, L., \& Shi, Y. (2018). Focal vibration stretches muscle fibers by producing muscle waves. IEEE Transactions on Neural Systems and Rehabilitation Engineering, 26(4), 839-846. https://ieeexplore.iee.org/document/8319449.

Hanson, M., \& Concialdi, M. (2019). Motor imagery in multiple sclerosis: exploring applications in therapeutic treatment. Journal of neurophysiology, 121(2), 347-349. https://pubmed.ncbi.nlm.nih.gov/30207860/.

Jordan, M. J., Norris, S. R., Smith, D. J., \& Herzog, W. (2005). Vibration training: an overview of the area, training consequences, and future considerations. The Journal of Strength \& Conditioning Research, 19(2), 459-466. https://pubmed.ncbi.nlm.nih.gov/15903391/.

Khalifeloo, M., Naghdi, S., Ansari, N. N., Akbari, M., Jalaie, S., Jannat, D., \& Hasson, S. (2018). A study on the immediate effects of plantar vibration on balance dysfunction in patients with stroke. Journal of exercise rehabilitation, 14(2), 259. https://www.ncbi.nlm.nih.gov/pmc/articles/PMC5931163/.

Kitazaki, S., \& Griffin, M. J. (1997). Resonance behaviour of the seated human body and effects of posture. Journal of biomechanics, 31(2), 143-149. https://www.sciencedirect.com/science/article/abs/pii/S0021929097001267.

Kojima, S., Onishi, H., Miyaguchi, S., Kotan, S., Sasaki, R., Nakagawa, M., ... \& Tamaki, H. (2018). Modulation of corticospinal excitability depends on the pattern of mechanical tactile stimulation. Neural plasticity, 2018. https://www.ncbi.nlm.nih.gov/pmc/articles/PMC5903327/.

Lacerda, N. N. D., Gomes, É. B., \& Pinheiro, H. A. (2013). Efeitos da facilitação neuromuscular proprioceptiva na estabilidade postural e risco de quedas em pacientes com sequela de acidente vascular encefálico: estudo piloto. Fisioterapia e Pesquisa, 20(1), 37-42. https://www.scielo.br/scielo.php?script=sci_arttext\&pid=S1809-29502013000100007.

Lakshminarayanan, K., Wang, F., Webster, J. G., \& Seo, N. J. (2017). Feasibility and usability of a wearable orthotic for stroke survivors with hand impairment. Disability and Rehabilitation: Assistive Technology, 12(2), 175-183. https://pubmed.ncbi.nlm.nih.gov/26735630/.

Li, W., Li, C., Xiang, Y., Ji, L., Hu, H., \& Liu, Y. (2019a). Study of the activation in sensorimotor cortex and topological properties of functional brain network following focal vibration on healthy subjects and subacute stroke patients: An EEG study. Brain research, 1722, 146338. https://pubmed.ncbi.nlm.nih.gov/31323197/.

Li, W., Li, C., Xu, Q., \& Ji, L. (2019b). Effects of focal vibration over upper limb muscles on the activation of sensorimotor cortex network: an EEG study. Journal of healthcare engineering, 2019. https://www.hindawi.com/journals/jhe/2019/9167028/.

Maceira-Elvira, P., Popa, T., Schmid, A. C., \& Hummel, F. C. (2019). Wearable technology in stroke rehabilitation: towards improved diagnosis and treatment of upper-limb motor impairment. Journal of neuroengineering and rehabilitation, 16(1), 1-18. https://www.ncbi.nlm.nih.gov/pmc/articles/PMC6862815/. 
Research, Society and Development, v. 10, n. 5, e13310514736, 2021

(CC BY 4.0) | ISSN 2525-3409 | DOI: http://dx.doi.org/10.33448/rsd-v10i5.14736

Martin, B. J., \& Park, H. S. (1997). Analysis of the tonic vibration reflex: influence of vibration variables on motor unit synchronization and fatigue. European journal of applied physiology and occupational physiology, 75(6), 504-511. https://pubmed.ncbi.nlm.nih.gov/9202946/.

Medeiros, C. S. P. D., Fernandes, S. G. G., Lopes, J. M., Cacho, E. W. A., \& Cacho, R. D. O. (2014). Efeito da terapia de espelho por meio de atividades funcionais e padrões motores na função do membro superior pós-acidente vascular encefálico. Fisioterapia e Pesquisa, 21(3), 264-270. https://www.scielo.br/scielo.php?pid=S1809-29502014000300264\&script=sci_arttext\&tlng=pt.

MINISTÉRIO DA SAÚDE. Censo Demográfico de 2020 e o mapeamento das pessoas com deficiência no Brasil. Departamento de Ações Programáticas e Estratégicas Coordenação Geral de Saúde da Pessoa com Deficiência. https://www2.camara.leg.br/atividade-legislativa/comissoes/comissoespermanentes/cpd/arquivos/cinthia-ministerio-da-saude.

Motta, E. M., \& Pereira, J. R. D. (2019). Estudo sobre Indicadores de Produção Científica Versus Produção Tecnológica na Universidade Estadual de Maringá. Cadernos de Prospecção, 12(4), 795. https://periodicos.ufba.br/index.php/nit/article/view/32767/20134.

Moura, M. E. B., Lago, E. C., Mesquita, G. V., \& Alves, E. L. M. (2014). Tendências e possibilidades do registro de marcas e patentes: análise reflexiva. Rev enferm UFPE on line, 8(1), 2494-7. DOI: 10.5205/reuol.5927-50900-1-SM.0807esp201438.

Mueller, S. P. M., \& Perucchi, V. (2014). Universidades e a produção de patentes: tópicos de interesse para o estudioso da informação tecnológica. Perspectivas em Ciência da Informação, 19(2), 15-36. https://www.scielo.br/pdf/pci/v19n2/03.pdf.

Paranhos, R. D. C. S., \& Ribeiro, N. M. (2018). Importância da prospecção tecnológica em base em patentes e seus objetivos da busca. Cadernos de Prospecção, 11(5), 1274. DOI: http://dx.doi.org/10.9771/cp.v12i5.28190.

Pola, H. M. C., Bergmann, K. E., \& Silva, J. S. (2015). Desempenho Funcional em Hemiparéticos Crônicos Antes e Após Programa de Exercício com Facilitação Neuromuscular Proprioceptiva. Revista Brasileira de Saúde Funcional, 2(1), 46-46. http://www.seer-adventista.com.br/ojs3/index.php/RBSF/article/view/663.

Pompeu, J. E., Alonso, T. H., Masson, I. B., Pompeu, S. M. A. A., \& Torriani-Pasin, C. (2014). Os efeitos da realidade virtual na reabilitação do acidente vascular encefálico: Uma revisão sistemática. Motricidade, 10(4), 111-122. http://www.scielo.mec.pt/pdf/mot/v10n4/v10n4a12.pdf.

Prabhu, D., Hasan, M. M., Wise, L., MacMahon, C., \& McCarthy, C. (2020, July). VibroSleeve: A wearable vibro-tactile feedback device for arm guidance. In 2020 42nd Annual International Conference of the IEEE Engineering in Medicine \& Biology Society (EMBC) (pp. 4909-4912). IEEE. https://ieeexplore.ieee.org/document/9176028.

Santos, M. D. M., Coelho, G. M., Santos, D. M. D., \& Fellows Filho, L. (2010). Prospecção de tecnologias de futuro: métodos, técnicas e abordagens. Parcerias estratégicas, 9(19), 189-230. http://seer.cgee.org.br/index.php/parcerias_estrategicas/article/view/253/247.

Seo, N. J., Woodbury, M. L., Bonilha, L., Ramakrishnan, V., Kautz, S. A., Downey, R. J., ... \& Vatinno, A. A. (2019). TheraBracelet stimulation during taskpractice therapy to improve upper extremity function after stroke: a pilot randomized controlled study. Physical therapy, 99(3), 319-328. https://academic.oup.com/ptj/article/99/3/319/5303730.

Souza, C. D., Barbastefano, R. G., \& Pereira, F. D. C. (2011). Estudo sobre o perfil dos inventores das patentes de universidades brasileiras. XXXI Encontro Nacional De Engenharia de Produção. Inovação Tecnológica e Propriedade Intelectual: Desafios da Engenharia de Produção na Con solidação do Brasil no Cenário Econômico Mundial Belo Horizonte, MG, Brasil, 4. http://www.abepro.org.br/biblioteca/enegep2011_TN_STO_142_896_18859.pdf.

Toscano, M., Celletti, C., Viganò, A., Altarocca, A., Giuliani, G., Jannini, T. B., ... \& Di Piero, V. (2019). Short-term effects of focal muscle vibration on motor recovery after acute stroke: a pilot randomized sham-controlled study. Frontiers in neurology, 10, 115. https://www.ncbi.nlm.nih.gov/pmc/articles/PMC6401608/.

Wei, W. X., Fong, K. N., Chung, R. C., Cheung, H. K., \& Chow, E. S. (2018). "Remind-to-move" for promoting upper extremity recovery using wearable devices in subacute stroke: a multi-center randomized controlled study. IEEE Transactions on Neural Systems and Rehabilitation Engineering, 27(1), 51-59. https://pubmed.ncbi.nlm.nih.gov/30475722/.

Zinke, F., Gebel, A., Granacher, U., \& Prieske, O. (2019). Acute effects of short-term local tendon vibration on plantar flexor torque, muscle contractile properties, neuromuscular and brain activity in young athletes. Journal of sports science \& medicine, 18(2), 327. https://pubmed.ncbi.nlm.nih.gov/31191103/. 